\title{
Scaling and Gingival Curettage A Radioautographic Study
}

\author{
BY S. STONE, * S. P. RAMFJORD* AND J. WALDRON,** \\ ANN ARBOR, MICHIGAN
}

SCALING WITH OR WITHOUT deliberate gingival curettage is used universally in periodontal therapy. Numerous ${ }^{1-8}$ studies have evaluated and attempted to perfect scaling techniques, but the periodontal reaction including healing and regeneration following such procedures has received less attention.

The purpose of the present investigation was to study in a sequential order the cellular dynamics leading to reestablishment of a physiologic gingival sulcus following scaling and light gingival curettage. With the advent of tritiated thymidine, an important tool has become available for studies of healing and regeneration. Since this isotope technique cannot be applied to human studies for safety reasons, experimental animals have to be used. It appears that some species of monkeys have periodontal tissues with biologic characteristics similar to humans. This investigation, therefore, was performed in rhesus monkeys, although there probably are some differences between monkeys and humans related to species and oral environment with regard to rate of healing and potential for regeneration. Thus this study represents an investigation of biologic principles rather than an evaluation of clinical procedures directly applicable to patients with periodontal disease.

\section{MATERIALS}

The present study is part of a radioautographic and histologic investigation which included healing of periodontal tissues both

*'The University of Michigan, School of Dentistry, Ann Arbor, Michigan.

**The Veterans Administration Hospital, Ann Arbor, Michigan. following gingivectomy and following scaling with curettage in rhesus monkeys. Three adult male rhesus monkeys, weighing respectively $11.8,9.1$ and $7.3 \mathrm{kgs}$, were used for the experiment. Adult males were selected in order to avoid possible fluctuations in mitotic rate associated with oestrus in females. ${ }^{9}$ All animals had a full complement of permanent teeth, including third molars. They had moderate to severe marginal gingivitis, with supra and subgingival calculus. The depth of the gingival crevice around the experimental teeth ranged from 2-4 $\mathrm{mm}$. with early beginning of periodontal pocket formation. Since there was considerable variation in the amount of calculus and degree of gingivitis, both in the same monkeys and from one monkey to another, all experimental teeth were scaled and polished superficially two weeks prior to the experiment. Complete removal of subgingival calculus was not attempted at this time. The scaling was done in an attempt to standardize the experimental conditions and included also the control teeth that were not to be treated later during the experimental procedures.

The preliminary scaling was done with \#2s, \#3s, and \#4s sickle scalers and \#5 and \#6 Bunting Curettest and \#6 and \#7 curettes. $\neq$ The crowns of the teeth were polished with flour of pumice in a polishing cup run by a dental engine. Only maxillary and mandibular central and lateral incisors were used for the experiment, since these teeth offered easy access and visibility to instrumentation as well as fair uniformity of gingival morphology and tooth contour.

fS. S. White Dental Supply Company.
\$The American Instrument Company.

Page 63/415 


\section{EXPERIMENTAL PROCEDURES}

Scaling and gingival curettage was carried out at intervals of respectively $2,5,9$, $13,25,49,73$ hours and 5, 9, 14, 21 and 35 days prior to the sacrifice of the animal. Each experimental procedure involved the buccal, lingual and interproximal surfaces of one anterior tooth. Six incisors were left untreated as controls. The control teeth were one each from the maxilla and the mandible of all of the experimental animals.

After the animals were anesthetized, the experimental teeth were thoroughly scaled with the previously listed instruments to assure complete removal of residual calculus. The scaling was followed by a gentle curettage of the soft tissue wall of the gingival sulcus with curettes \#6 and \# 7.* No distinct attempt was made to remove all of the crevicular and attachment epithelium. The scaling and the curettage were limited to the bottom of the epithelial attachment as located by definite resistance to the instruments. No lavaging or aspiration was used during or after the instrumentation and blood clots were allowed to form undisturbed in the treated crevices. The experimental procedures were equally divided between two investigators.

On the day each monkey was scheduled to be sacrificed one microcurie of tritiated thymidine (specific activity 6.7 curies per millimole)** per gram of body weight was administered intravenously at 10:00 a.m. One hour later (11:00 a.m.), the animals were sacrificed by ex-sanguination. This time schedule was kept in order to avoid possible mitotic influence associated with the diurinal cycle. ${ }^{10}$

The jaws were dissected out and placed in $10 \%$ buffered neutral formalin fixative for three weeks, segmented into blocks by a rotary diamond disc and decalcified in $20 \%$ ethylene diamine tetraacetic acid

* The American Instrument Company.

**The New England Nuclear Corporation, Boston Massachusetts.

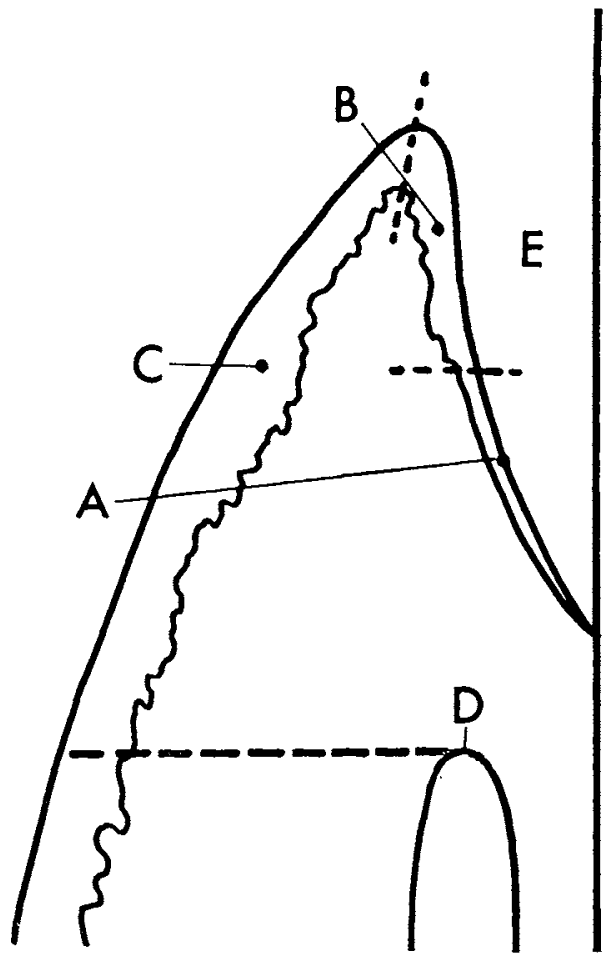

Fig. 1. Diagram demonstrates the three reference zones. " $A$ " indicates the epithelial attachment; " $B$ " the crevicular epithelium; " $C$ " the surface gingival epithelium; "D" enamel; " $\mathrm{E}$ " alveolar crest.

(E.D.T.A.) which had been adjusted to a $\mathrm{pH}$ of 7.4 with sodium hydroxide. Following decalcification, the specimens were embedded in paraffin and sectioned at $8 \mathrm{mi}-$ crons.

Specimens from internal organs also were secured and studied microscopically to assure that the monkeys were not afflicted with any serious systemic disorders which might have affected the healing of the periodontal structures.

A large number of radioautographs were prepared according to the technique described in previous publications. ${ }^{11}$ Most sections were stained with hematoxylin only in order to facilitate radioautographic identification and cell counting; however, histological sections without superimposed radioautographs were stained with regular hematoxylin and eosin stains. 


\section{PROCEDURES FOR EVALUATION OF} THE RADIOAUTOGRAPHS

In order to facilitate the radioautographic evaluation, the gingival epithelium was divided into three zones, in a fashion similar but not identical to previous studies. ${ }^{11,12}$ Figure 1 indicates these three zones which in the remainder of the paper will be referred to as Zones A, B and C. Zone A corresponds to the epithelial attachment or approximately the apical one-half of the gingival sulcus. Zone B extends coronally from the epithelial attachment to the crest of the free gingiva. When the typical morphologic boundary between Zones A and B was obscured in specimens obtained shortly after the curettement or as a result of massive infiltration of inflammatory cells, an arbitrary boundary was set in the middle of the crevice. This, however, had very little if any influence on the end result since in these cases there were practically no epithelial cells left to be counted in this boundary area. Zone $\mathrm{C}$ comprised the gingival surface epithelium from the free gingival margin to the level of the crest of the alveolar process. In some instances only sections from the interproximal area were available, and in these cases Zone $\mathrm{C}$ could not be counted.

Since various amounts of surface epithelium had been removed by the experimental procedures, the usual counting and computations of radioactivity indices (as outlined by Messier and Leblond ${ }^{13}$ with counting of the total number of labeled and unlabeled cells in microscopic fields of epithelium) would have given a distorted index related to the amount of surface epithelium that had been removed. It was therefore decided to count only the basal cells in all three zones and relate the number of labeled basal cells to the total number of basal cells for each zone. This provided a radioactivity index (R.A.I.) or percentage of labeling different from Messier and Leblond's ${ }^{13}$ radioactivity index, but more comparable as a basis for study of variations in premitotic activity between the three zones. Radioautographs from the control specimens were evaluated in the same manner.
The total number of basal epithelial cells was first counted in one of the zones using a Veeder* hand tally counter, then the number of labeled basal epithelial cells was determined for that same zone. The counts were done at X430 magnification. Heavy inflammatory infiltrations, in sections from short term experimental periods, sometimes made it difficult to identify the basal cells of the epithelium. In such instances counts were made 2 or 3 times at intervals varying from one to several weeks in order to assess if a possible error in identification and counting had influenced the results. No significant discrepancies existed between these different countings. The radioactivity indexes were identical or very close to an earlier or subsequent figure, so this source of error could be ruled out. An attempt was made to count an equal number of sections from the same experimental period. A mean (X) R.A.I. was computed for each zone on each slide, then the mean of the means of all slides ( $\overline{\mathrm{X}} \mathrm{T})$ for each zone was determined. A summary of the cell counts and radioactivity indices appears in Table 1.

\section{FINDINGS}

The uptake of tritiated thymidine is almost completed within 10-15 minutes after intravenous injections. ${ }^{14,}{ }^{15}$ In order to obtain a radiographic recording of the cellular activity, for example, one hour after the curettage, the isotope was administered one hour after the curettage; but the animal was killed one hour later in order to secure full uptake and standardize procedures. The histological findings, therefore, are from approximately one hour longer interval of experimental time than the radioautographs. In the description of the findings and legends for the photomicrographs of short term specimens both histologic and radioautographic time intervals have been indicated. The descriptions are all made with reference to Zones A, B and C as indicated in Fig. 1.

*Veeder Root, Inc., Hartford, Connecticut. 
TABLE 1

Cell Counts and Radioactive Indices (R.A.I.)

\begin{tabular}{|c|c|c|c|c|c|c|c|c|c|}
\hline \multirow[b]{2}{*}{$\begin{array}{c}\text { Exp. } \\
\text { Interval }\end{array}$} & \multicolumn{3}{|c|}{ Zone $A$} & \multicolumn{3}{|c|}{ Zone B } & \multicolumn{3}{|c|}{ Zone C } \\
\hline & $\begin{array}{c}\text { Total } \\
\text { Cells } \\
\text { Counted }\end{array}$ & $\begin{array}{l}\text { Labeled } \\
\text { Cells } \\
\text { Counted }\end{array}$ & R.A.I. & $\begin{array}{c}\text { Total } \\
\text { Cells } \\
\text { Counted }\end{array}$ & $\begin{array}{l}\text { Labeled } \\
\text { Cells } \\
\text { Counted }\end{array}$ & & $\begin{array}{c}\text { Total } \\
\text { Cells } \\
\text { Counted }\end{array}$ & $\begin{array}{l}\text { Labeled } \\
\text { Cells } \\
\text { Counted }\end{array}$ & R.A.I. \\
\hline 1 hour & 1,630 & 61 & .037 & 1,873 & 164 & .088 & 12,570 & 842 & .067 \\
\hline 4 hours & 1,226 & 48 & .039 & 10,932 & 680 & .062 & & - & - \\
\hline 8 hours & 1,858 & 19 & .012 & 2,078 & 105 & .051 & 16,434 & 561 & .034 \\
\hline 12 hours & 1,026 & 09 & .009 & $\ldots$ & $\ldots$ & — & 12,286 & 530 & .043 \\
\hline 24 hours & 835 & 282 & .338 & 1,571 & 455 & .290 & 12,758 & 1,619 & .176 \\
\hline 36 hours & 2,238 & 525 & .235 & 1,658 & 454 & .274 & 10,877 & 1,051 & .097 \\
\hline 48 hours & 439 & 50 & .114 & 812 & 97 & .119 & 2,729 & 212 & .078 \\
\hline 3 days & 448 & 11 & .025 & 2,244 & 235 & .105 & - & - & - \\
\hline 5 days & 1,182 & 34 & .029 & 3,680 & 410 & .111 & 12,884 & 482 & .037 \\
\hline 9 days & 1,132 & 129 & .114 & 1,487 & 162 & .109 & & - & \\
\hline 14 days & 2,433 & 48 & .020 & 3,864 & 449 & .116 & 8,088 & 537 & .066 \\
\hline 21 days & 1,166 & 43 & .037 & 2,382 & 283 & .118 & 8,877 & 386 & .043 \\
\hline 35 days & 1,897 & 47 & .026 & 2,359 & 346 & .147 & 4,333 & 818 & .022 \\
\hline Six Control & & & & & & & & & \\
\hline Teeth & 6,533 & 517 & .079 & 11,396 & 1,679 & .147 & 35,462 & 1,780 & .050 \\
\hline
\end{tabular}

Histopathologic findings two hours after curettage indicated that part of the epithelial attachment had been retained during the scaling and curettage (Fig. 2A). There were some tears within the residual epithelium that also extended into the gingival fibers apically to the epithelial attachment. Epithelial cells, coagulated blood and cellular debris were found adherent to the root surface and in the crevicular space. Numerous polymorphonuclear leukocytes were present between the residual epithelial cells and on the surface bordering the crevice, both in Zones A and B (Fig. 2B).

The only epithelium retained in Zone B was found at the middle third. There was dilatation of blood vessels, edema, aggregation of polymorphonuclear cells toward the surface and slight alteration of the morphology of the connective tissue. Small bits of previously calcified debris were adherent to the surface of the crevicular wound.

There were few labeled epithelial cells within Zones $\mathrm{A}$ and $\mathrm{B}$ and the radioactive indices were low (see Table 1). At the junction of Zones $\mathrm{B}$ and $\mathrm{C}$ (the free gingival margin) and bordering the wound there was an area of necrotic epithelium with loss of the normal arrangement of epithelial cells, decreased staining potential and no labeling. The rest of the epithelium in Zone $\mathrm{C}$ appeared undisturbed and the labeling corresponded to labeling of similar control areas (see Table 1). There were a few labeled cells in the connective tissue associated with areas of chronic inflammation which apparently had been present before the scaling and curettage.

The five hour specimen was from an interproximal area sectioned mesio-distally so there was no Zone C. The epithelial attachment of Zone A had been partially retained, and at the apical third of Zone $\mathbf{A}$, epithelial cells were seen adhering to the root surface. The surface cells of Zone $B$ had been removed. Long, thin rete pegs extended into an area of chronic inflammation. It appeared that these rete pegs and the chronic inflammation had been present prior to the scaling.

Polymorphonuclear leukocytes were found in the connective tissue adjoining the 


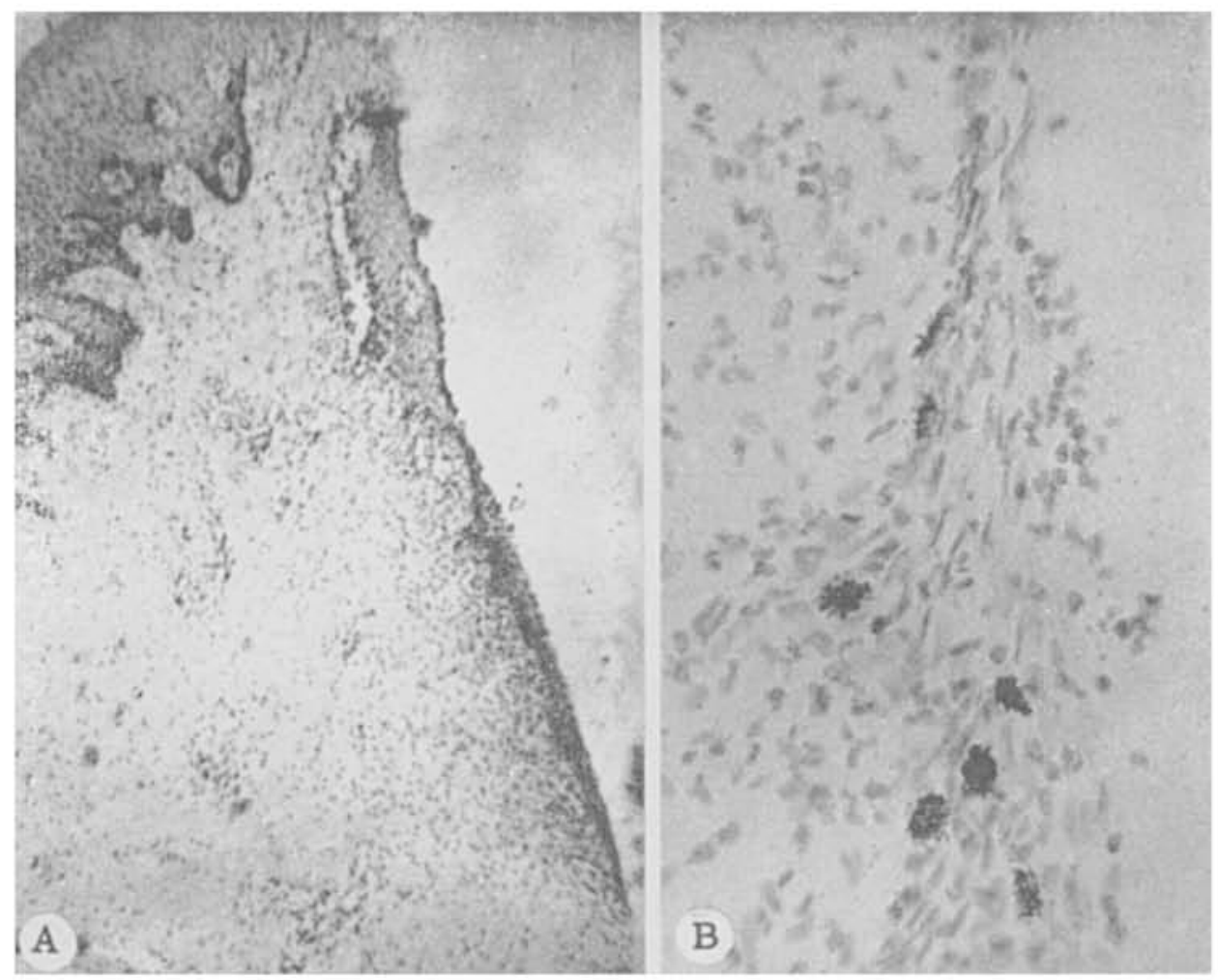

Fig. 2A. Two hours after curettage. Thin discontinuous layer of remaining crevicular and attachment epithelium. Moderate labeling of epithelial cells in all of the three experimental zones one hour after the curettage. (Magnification X43). Fig. 2B. High magnification from Zone B at the border of Zone A from Fig. 2A. Disruption of continuity of surface epithelium. Polymorphonuclear cells on the surface, between the epithelial cells and in the subepithelial connective tissue. Labeling of basal and prickle cells. (Magnification X525)

crevice, throughout the retained epithelium and in the crevicular space. There was extensive dilation of the capillary vessels which contained numerous polymorphonuclear leukocytes. In the deeper areas of the connective tissue there was chronic inflammation which appeared to have been there prior to the curettage. Cellular debris, pieces of calculus and fragments of blood clots were present in the crevice.

There were labeled cells only occasionally in Zones A and B, with some of the labeling in Zone B appearing close to the gingival margin. Only a few labeled cells were seen in the underlying connective tissues associated with areas of chronic inflammation.

In the nine hour specimen, the epithelial attachment of Zone A was partially removed, mostly towards the junction of Zone
B (Fig. 3A). Part of the epithelium also was removed in Zone B leaving only 2-3 layers of cells lining the crevice and some rete pegs extending into the connective tissue (Fig. 3B). Most of the coronal third of the crevicular epithelium was retained. Polymorphonuclear leukocytes were found adjacent to and infiltrated into Zone B. Polymorphonuclear leukocytes also appeared between the residual epithelial cells and in the crevice corresponding to Zone A. Adjacent to the apical end of Zone A there were some plasma cells. Some cellular debris and remains of blood clot were present in the gingival crevice. Labeling in Zone A was minimal (see Table 1). The R.A.I. only was a third of the R.A.I. found in the one and four hour specimens. The labeling in both Zones $\mathrm{B}$ and $\mathrm{C}$ was most prominent at the gingival crest. 

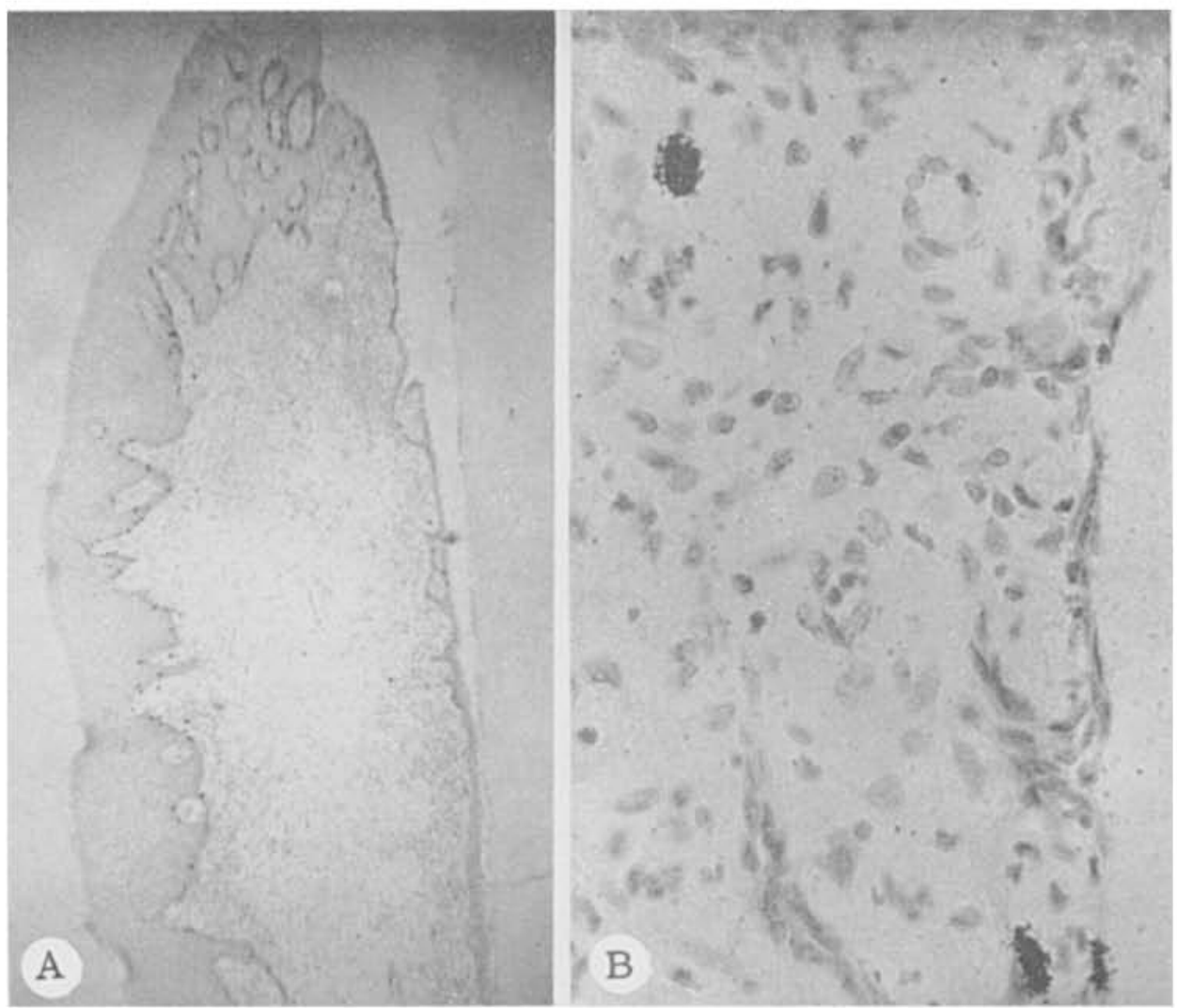

Fig. 3A. Nine hours after curettage. Some residual epithelium both in Zones A and B. Fig. 3B. High magnification from Zone B, Fig. 3A. Retained epithelial pegs with minimal labeling. Dilated capillaries (A). (Magnification X525)

In the thirteen hour specimen, the epithelium in both Zones A and B had been almost completely removed (Fig. 4A). Only a few epithelial cells remained at the apical third of Zone A (Fig. 4B). Polymorphonuclear leukocytes were seen within the residual epithelium and along the root surface corresponding to Zone A. At Zone B some connective tissue apparently had been removed during the instrumentation. The wound was covered by dense masses of fibrin polymorphonuclear leukocytes and fragmented cellular debris.

The connective tissues underlying Zones A and B demonstrated marked capillary dilatation with some decrease in the number of young fibroblasts compared with the shorter term specimens. There was edema and disruption of the fibrous pattern of the connective tissue immediately under the wound surface. A minimal labeling of the retained cells of Zone A was observed and no labeling was seen adjacent to the area of necrosis at the gingival crest. In Zone $\mathrm{C}$ the R.A.I. was similar to the control specimens.

Most of the epithelium of Zone A apparently had been removed during the curettage in the twenty-five hour specimen, and almost all of the remaining epithelial cells were basal cells. There was a marked influx of polymorphonuclear cells, both between the remaining epithelial cells and on the surface of the curetted area. Some epithelium remained in Zone B, but it showed marked intracellular edema and a large number of polymorphonuclear cells covered the crevicular surface. At the free gingival margin of Zone $\mathrm{C}$, there was necrosis and some disruption in the normal pattern of the epithelium (Fig. 5). 


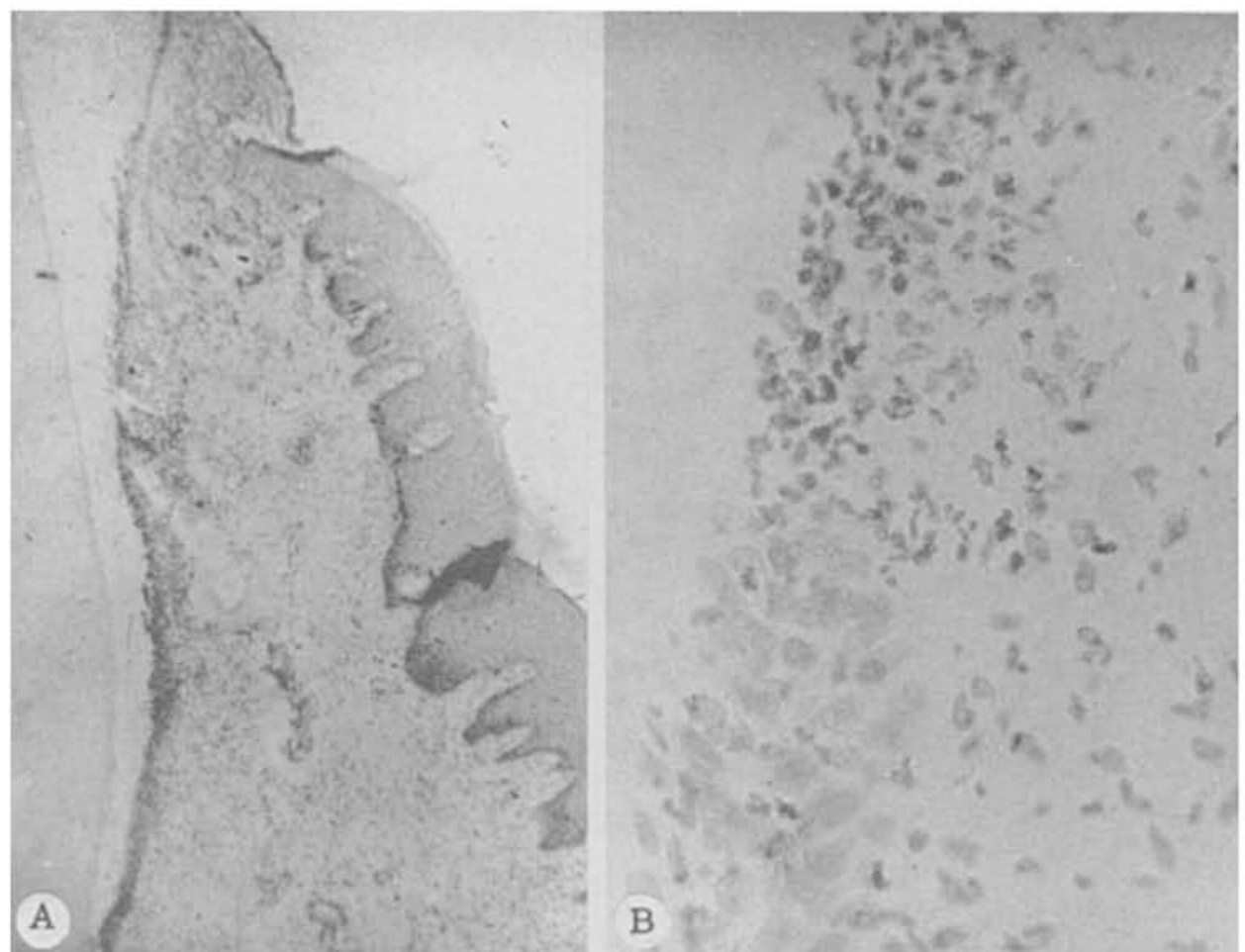

Fig. 4A. Thirteen hours after curettage. The epithelium has been almost completely removed except at the apical aspect of Zone A. Fig. 4B. High magnification from apical aspect of Zone A, Fig. 4A. Residual epithelium at the bottom of the photomicrograph. No labeling in the residual epithelium. (Magnification X525)

Radioautographs 24 hours after the curettage showed wide spread and intense labeling of the epithelial cells in all zones. The

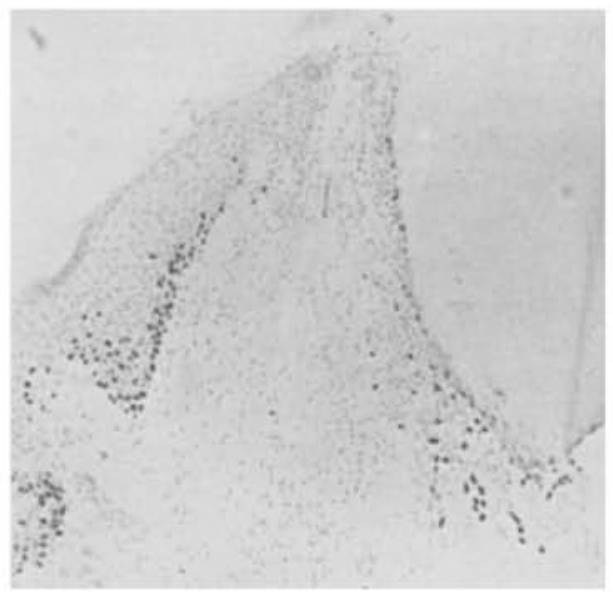

Fig. 5. Twenty-five hours after curettage. Labeling 24 hours after curettage. Only a few basal cells remaining of the epithelial attachment and the crevicular lining. Intensive labeling of remaining epithelial cells except at border of necrotic wound at free gingival margin. Also some labeling of connective tissue cells adjacent to gingival crevice. (Magnification X86) radioactive index both for Zones A and B had increased very much from the 12 hour radioautographs (see Table 1). At the free gingival margin of Zone $\mathrm{C}$ there was no labeling of the epithelium that bordered the wound. This lack of labeling corresponded to a reduction in the number of cells and poor staining qualities. But apically to this crestal necrobiotic area in Zone $\mathrm{C}$ the labeling was intense and included both basal and spinous cells. Slight increase in connective tissue labeling compared with the shorter term specimens occurred under the surface of Zone A.

Thirty-seven hours after curettage, the epithelium appeared continuous from the free gingival margin of Zone $\mathrm{B}$ to the apical base of Zone A (Fig. 6A).

Five to ten layers of epithelial cells lined the sulcus. The nuclei were pale staining and with prominent intercellular spaces. Poly- 


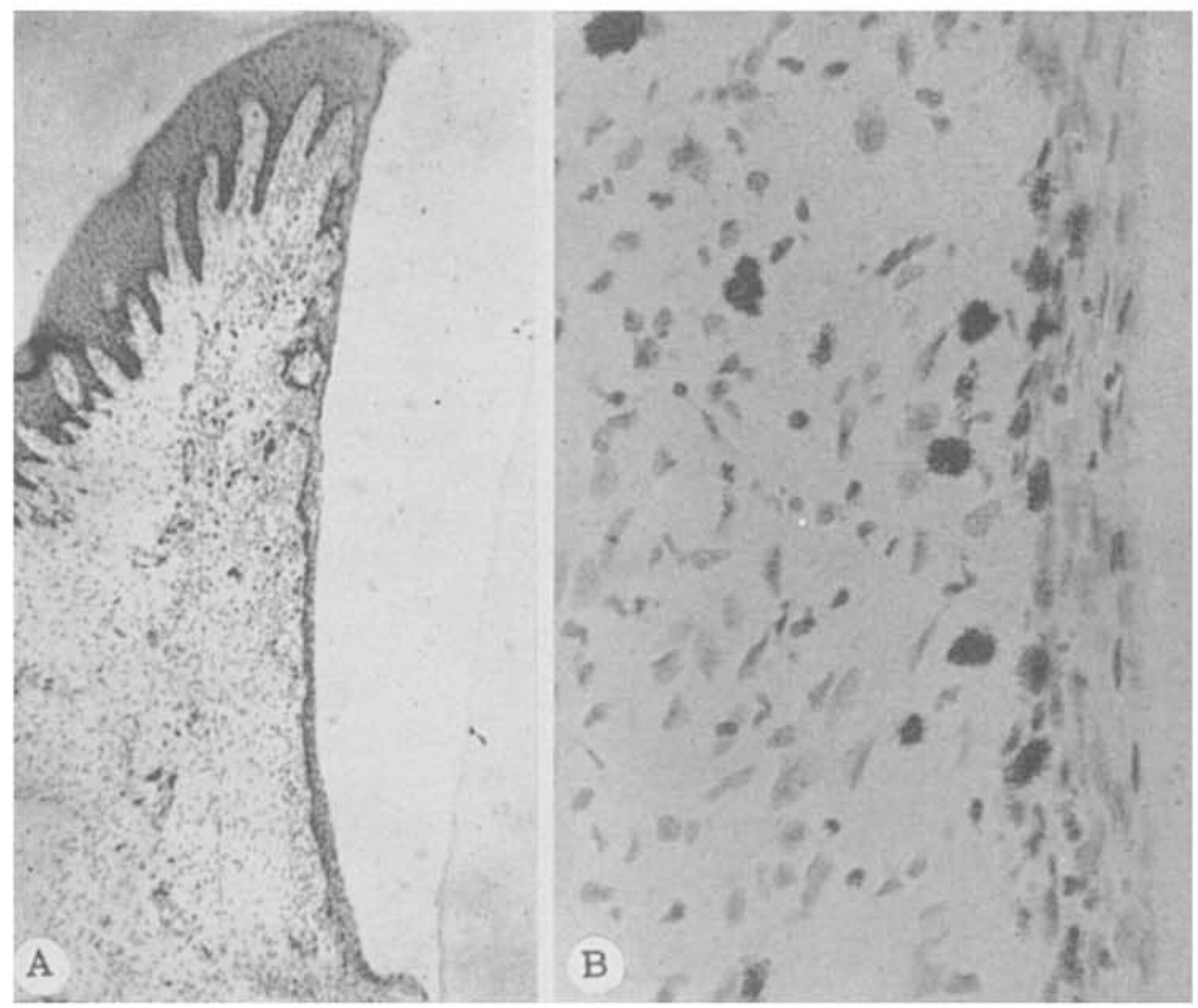

Fig. 6A. Thirty-seven hours after curettage. Labeled 36 hours after curettage. Continuity of crevicular and attachment epithelium. Labeling along the entire length of both Zones A and B. (Magnification X40). Fig. 6B. High magnification from Zone A, Fig. 6A. Labeling prominent in the connective tissue as well as in the basal cells of the epithelium. (Magnification X550)

morphonuclear leukocytes were spread diffusely throughout the epithelium and a few were scattered in the lamina propria. Most of the cells of Zone B apparently had regenerated after the experimental procedure, but there were some tears within the epithelium indicating that some cells had been there since the time of the curettage and trauma.

Labeled cells were seen throughout the basal layers of the epithelium both in Zones $\mathrm{A}$ and $\mathrm{B}$, and there was considerable labeling in the connective tissues adjacent to Zones $\mathrm{A}$ and B (Fig. 6B).

In the forty-nine hour specimen the epithelium of Zone A appeared to be separated from the tooth and only a few cells adhered to the corresponding root surface. The epithelial surface of Zone B was irregular and there was a large number of polymorphonuclear leukocytes in this Zone. There was also an increase in fibroblasts adjacent to Zones $\mathrm{A}$ and $\mathrm{B}$ compared to the control specimens. A few pieces of calculus were observed on the wound surface. There was labeling of basal cells along the entire length of Zones A and B (Fig. 7). Plasma cells and lymphocytes were seen associated with the most pronounced labeling in Zone A, while polymorphonuclear leukocytes accompanied the most pronounced labeling in Zone B.

Three days after curettage, the continuity of Zones A and B was evident as a well demarcated epithelial layer. The apical end of Zone A tapered into a single cell thickness. The epithelial cells at the apical end of Zone A were large, pale staining and had granular nuclei. The peripheral surface of both Zones 


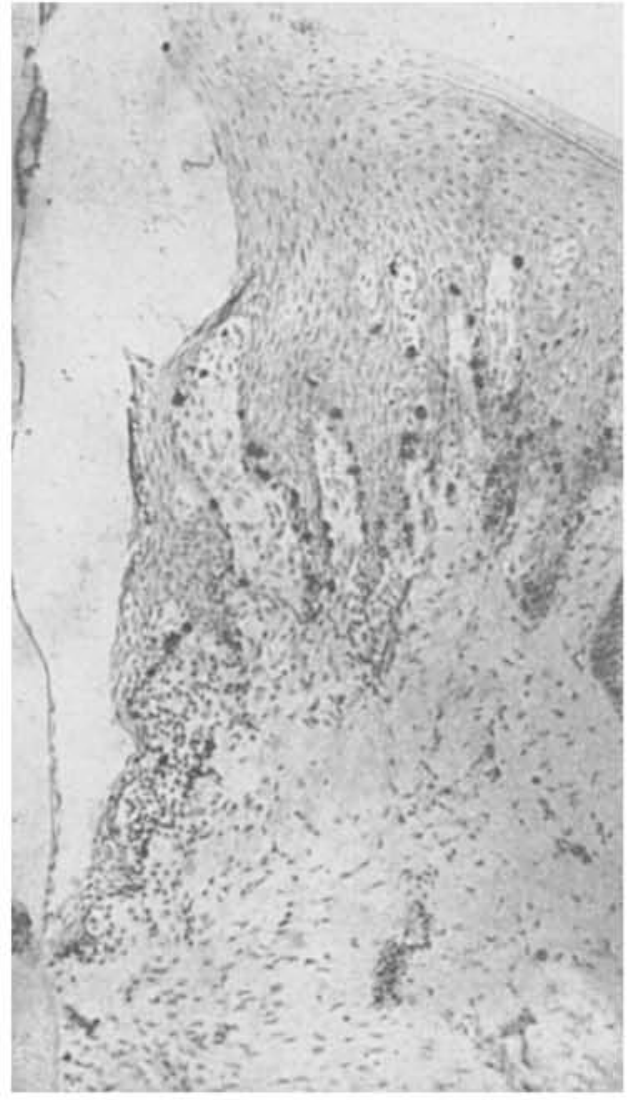

Fig. 7. Forty-nine hours after curettage. Labeled 48 hours after curettage. Labeling of basal epithelium in all zones. Connective tissue labeling mainly associated with inflammation. (Magnification X80)

A and B was intact. Occasional labeling was found in the epithelial attachment (Zone A), but the radioactivity index was considerably reduced compared to the one and two day specimens (see Table 1).

Connective tissue labeling was seen in lamina propria related to localized foci of inflammatory cells. The number of labeled fibroblasts seemed to be somewhat less than observed in the one and one-half and two day specimens.

Five days after curettage, the bottom part of the epithelial attachment in Zone A appeared normal and adherent to the tooth surface (Fig. 8). Zone B had a thick epithelial coverage. There were lymphocytes

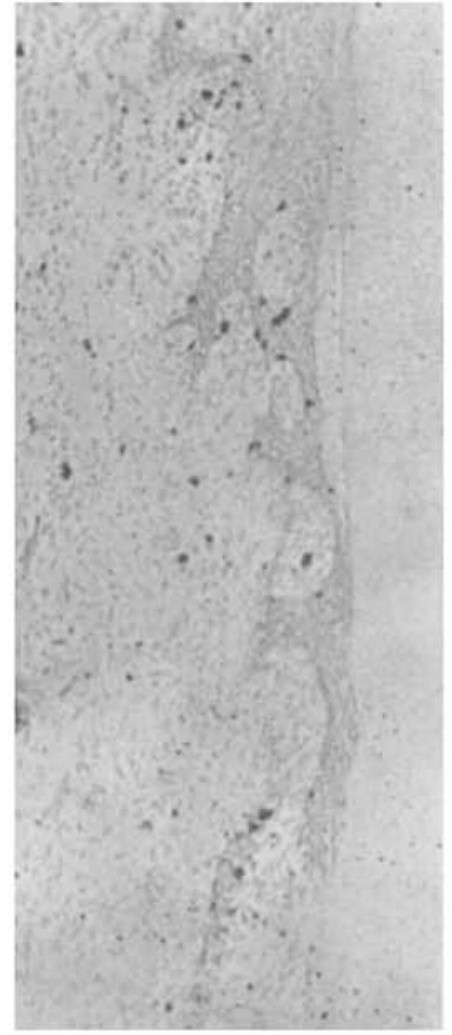

Fig. 8. Five days after curettage. Close adaptation or adherence of bottom of epithelial attachment to tooth surface No epithelial labeling in this attached area, but some labeling both in epithelium and connective tissue in the rest of the specimen. (Magnification X95)

and plasma cells spread throughout the lamina propria. Labeling of the epithelium was not prominent in any zone. The few labeled cells in Zone A appeared at the most coronal aspect of the epithelial attachment. More labeling was seen in Zone B than in Zone A especially toward the gingival margin (see Table 1).

The nine day specimen was from an interproximal area with intense inflammation. There was intercellular edema and prominent infiltration of polymorphonuclear leukocytes throughout Zones A and B. The epithelium in Zone B was irregular in thickness with deep rete peg projections into areas of underlying inflammation. The inflammatory cells in the deeper area were mainly lympho- 


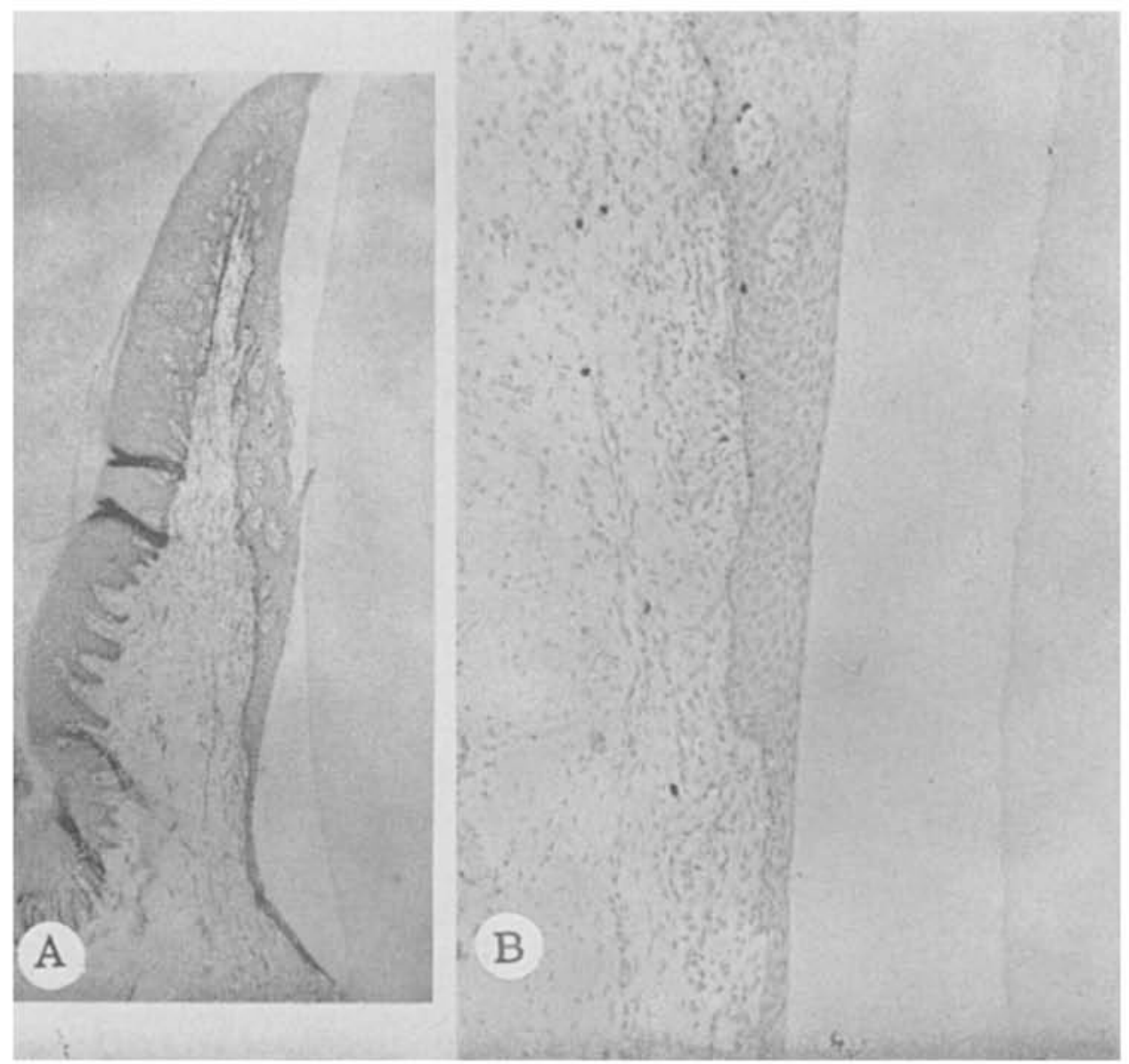

Fig. 9A. Fourteen days after curettage. Almost normal gingiva with minimal labeling in Zone A and slightly more in Zone B. (Magnification X32). Fig. 9B. High magnification from 9A. Junction between Zones A and B. Well defined epithelium with a few labeled cells in Zone B (upper two thirds of picture). A few labeled cells in connective tissue associated with mild chronic inflammation. (Magnification X95)

cytes. There was intensive labeling associated with the inflammation. At the apical aspect of Zone A there was minimal labeling. It should be noted that adjacent to this experimental tooth was a control tooth with residual calculus, this may at least in part have been responsible for the severe papillary inflammation.

Two weeks after the experimental procedure, the epithelial attachment in Zone A appeared well defined with tapering to a single cell layer at the bottom (Fig. 9A). Zone B also appeared continuous. Occasional inflammatory cells; plasma cells and lymphocytes were seen in a stroma of well defined mature fibrocytes. A distinct pattern of gingival fibers could be identified. There was very little labeling in Zone A with a little more labeling in the coronal part of Zone B corresponding to a slight chronic inflammation (Fig. 9B) (see Table 1).

Three weeks after scaling and curettage, the epithelial attachment of Zone A was long, narrow and well defined, tapering to a very thin attachment at the cemento-enamel junction. At the coronal aspect of Zone B the epithelial basal layer become less distinct. Plasma cells, lymphocytes and polymorphonuclear leukocytes were present in increasing number toward the free gingival margin. Polymorphonuclear leukocytes and cellular debris lined the crevicular surface. 


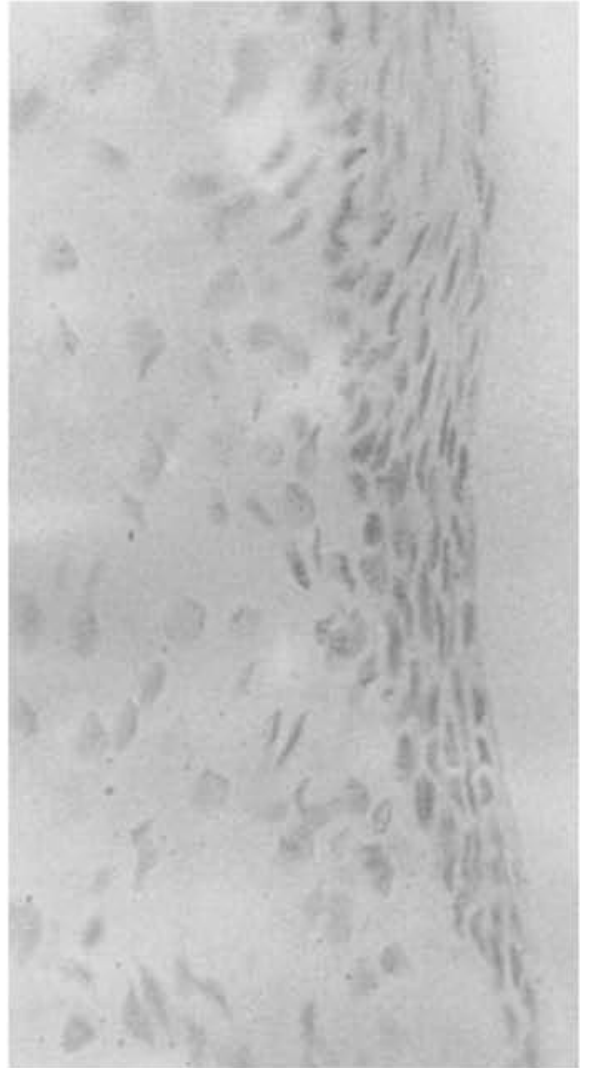

Fig. 10. Bottom of epithelial attachment 21 days after curettage. No labeling. (Magnification X525)

A few inflammatory cells extended toward the free gingival margin of Zone C.

The slight labeling of Zone A was confined to the coronal one-third of the epithelial attachment, and this labeling was associated with a loss of sharpness of definition of the basal cells. There was some labeling in Zone B corresponding to areas of inflammation (see Table 1). No labeling was found at the bottom of the epithelial attachment (Fig. 10).

Five weeks after scaling and curettage, the cells of the epithelial attachment were well adapted to the cementum (Fig. 11A). A well defined separation appeared between Zone A and Zone B with some inflammatory reaction adjacent to Zone $\mathrm{B}$. The few labeled cells in Zone A were limited in location to the border of Zone B (Fig. 11B). Zone A had a very low radioactive index $.030 \mathrm{com}$ pared with .143 in Zone B (Table 1). Labeling of the basal cells in Zone B was widely distributed along the length of the entire crevice (Fig. 11C). Premitotic activity indicated by labeling in the connective tissue was sparse and limited to perivascular regions.

Findings in Control Specimens. There was considerable inflammation in the gingival tissues of all of the control specimens with some variation in the extent and type of inflammatory cells. The epithelial attachment of Zone A did not appear to be well adapted to the tooth surface in most instances, and there was a wide variation in thickness of the epithelium of Zone B. Infiltration of polymorphonuclear cells into the epithelial tissues was seen often associated with inter and intraepithelial vacuolization and edema. In all instances there was some labeling in Zone A with most labeling associated with areas of extensive inflammatory reaction. The average labeling in Zone B, however, was considerably higher than in Zone A. The labeling in Zone A varied greatly from R.A.I. of .12 to .04. In these untreated cases the R.A.I. in Zone $\mathrm{C}$ was actually less than the index for Zone A (see Table 1). While following scaling and curettage, the R.A.I. 2 to 5 weeks after the treatment was considerably lower for Zone $\mathrm{A}$ than for Zone $\mathrm{C}$ (see Table 1).

Discussion of Findings. The significance of the histopathologic findings and the radioautographic findings will be discussed separately, but summarized together.

Tissue injury in the gingival crevice extended to the base of the epithelial attachment as reported previously for scaling with or without curettage.1, 4, 6 In some instances the traumatic effect was limited to slight tears of the epithelial tissues, while in other cases the tears also involved gingival fibers although an attempt had been made to restrict the apical extension of the scaling and curettage to the bottom of the epithelial attachment as felt by definite resistance to 

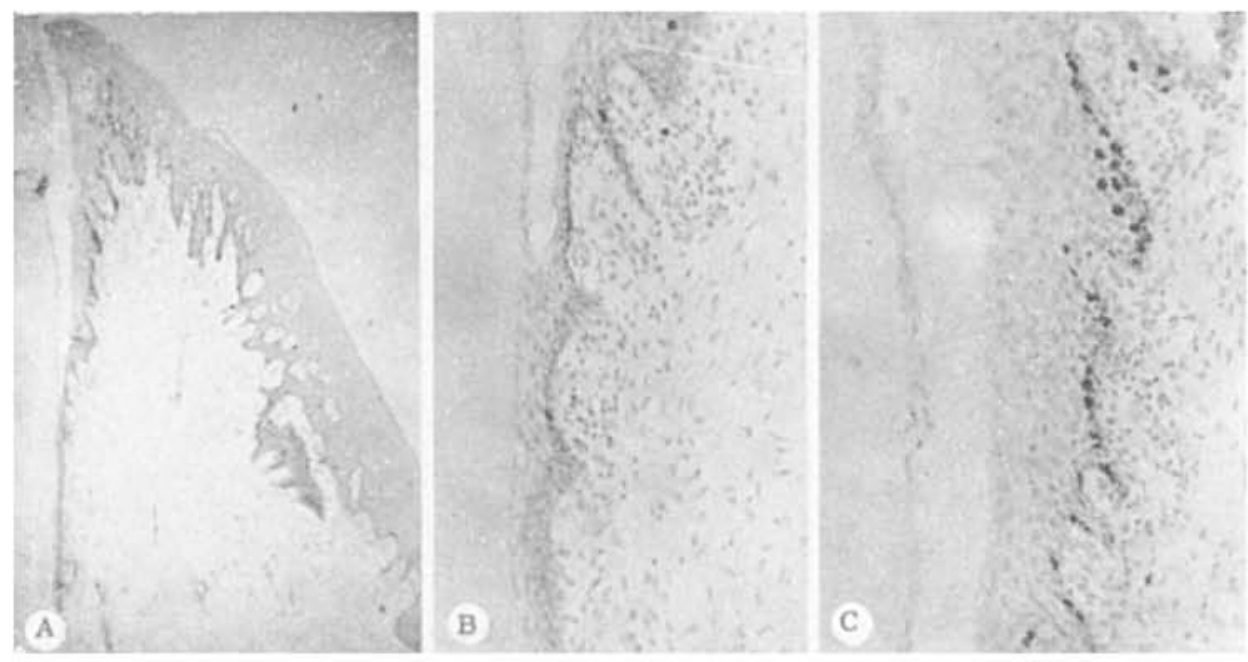

Fig. 11A. Thirty-five days after curettage. Inflammation at coronal aspect of Zone B. Long thin epithelial attachment. (Magnification X35). Fig. 11B. From Fig. 11A, junction between Zones A and B. Labeling decreasing in apical direction (toward bottom of picture). (Magnification X172). Fig. 11C. From Fig. 11A. Middle part of Zone $\mathrm{B}$ with considerab!e labeling and mild chronic inflammation. (Magnification X172)

the scaler. The tear of gingival and some periodontal fibers by these careful procedures should emphasize the need for careful light instrumentation at the bottom of any gingival crevice.

An early inflammatory reaction to scaling and curettage was manifested as dilation of vessels and a marked infiltration of polymorphonuclear leukocytes into the crevicular tissues and the crevice within two hours after the scaling. Polymorphonuclear cells were situated in the surface area and incorporated in the blood clot on the wound. The arrangement of these inflammatory cells relative to the crevicular wound resembled the description of Viziam, et al. ${ }^{16}$ of a "polyband" in curaneous wounds in rabbits. They assumed that the function of the polymorphonuclear cells was to protect the underlying tissues from bacterial invasion during the early healing process. There were numerous polymorphonuclear cells within the intercellular spaces of the remaining epithelium as observed by O'Bannon. ${ }^{5}$ This probably is part of the "poly-band" defense mechanism.

Calculus and tooth debris within the crevice and on the wound surface following scaling has been reported by many investigators ${ }^{1,4,6-8}$ and a similar observation in this study emphasizes the need for irrigation of the pockets following scaling.

The most common location for residual epithelium after curettage was at the apical base of Zone A. This agrees with the findings of some investigators, ${ }^{7,8,17}$ although others have found that most epithelium remained at the free gingival margin following scaling or curettage. ${ }^{1,3}$ However, one of these latter investigators ${ }^{3}$ attempted to place his curette $2 \mathrm{~mm}$. apically to the clinical sulcus; while in the present investigation the intent was not to go beyond the clinical sulcus, as indicated by resistance to the curette and it can be expected that going beyond the clinical sulcus with the curettes will assure more complete removal of the epithelial attachment than stopping at the first definite resistance. O'Bannon's ${ }^{5}$ assumption that the changes within the sulcus after scaling and curettage would reflect the approach of the operator was confirmed since the extent of the epithelial removal varied consistently between the two operators who performed the curettage. One of the operators had performed considerably more vigorous curettage than the other. The morphology of the 
EPITHELIAL PREMITOOTIC ACTIVITY AFTER SCALING AND CURETTAGE

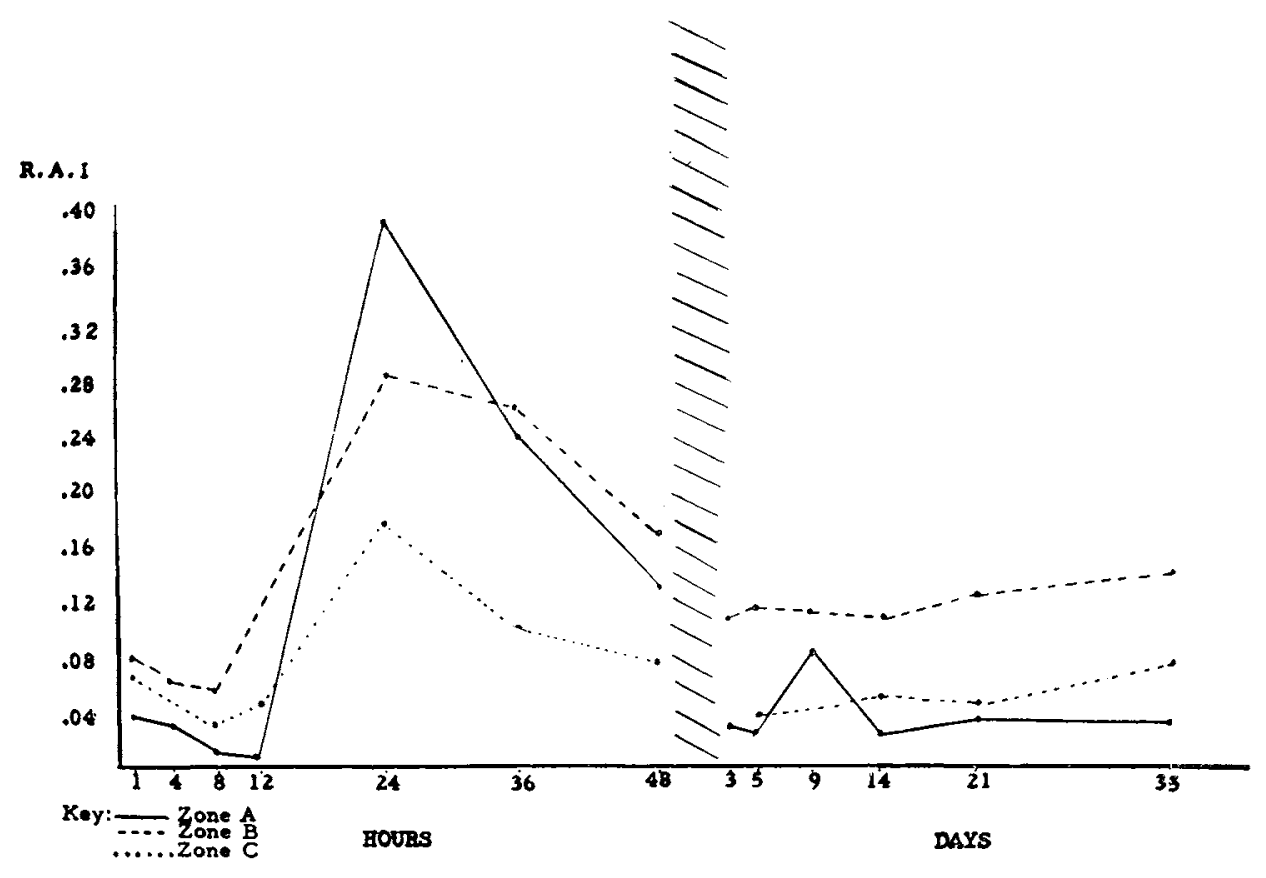

Fig. 12. Diagram of the epithelial premitotic activity.

teeth and the surrounding structures in monkeys are considerably different from humans, and the instruments that were used were designed for use in humans. Clinical features regarding efficiency of scaling and extent of curettage, therefore, are not entirely comparable.

Continuity of the sulcus epithelium from the coronal edge of Zone $B$ to the apical end of Zone A, first was observed in the thirtyseven hour specimen. Most investigators $1,5,8$ have found that longer time is required in humans and no conclusions can be made from this single case in a monkey, since the result certainly is related to the amount of curettage, the depth of the sulcus and the trauma from the curettage. More significant was the establishment of a normal epithelial attachment five days after the curettage, which is much shorter time than in our gingivectomy studies. 18,19

The inflammatory reaction at the gingival crest in the twenty-one day and thirty-five day specimen, was well correlated with the recurrence of plaque and beginning calculus formation in these cases. The entire Zone A and the apical aspect of Zone B, however, appeared to be adherent to the tooth in the thirty-five day specimen and the new inflammatory reaction had not penetrated to these areas five weeks after curettage, in spite of complete lack of oral hygiene.

Significance of Autoradiographic Findings. The labeling indicated that premitotic activity of the epithelial cells in Zones $A$ and $B$ declined from 1 hour to 12 hours after scaling and curettage (Table 1, Fig. 12). The R.A.I. in Zone $\mathrm{C}$ was also slightly reduced during this period but to a lesser extent. This depression of DNA synthesis was probably due to alteration of cellular metabolism subsequent to the trauma of scaling and curettage. Such a depression of activity, may as Bullough ${ }^{20}$ suggested, be based on a reduced cellular glucose level, which would deprive the cells of the energy needed to sustain mitosis. 
Page 76/428 Stone, RamFJord, Waldron

Twenty-four hours after scaling and curettage the premitotic activity in Zone $\mathrm{A}$ had increased 40 times when compared to the 12 hour sample (Fig. 12). It could not be determined from the present investigation when this rise in premitotic activity actually began during the interval of from 12 to 24 hours after the experimental procedures. Neither do we know whether it was a gradual or sudden increase.

The peak premitotic activity in Zones B and $\mathrm{C}$ also was recorded at the 24 hour interval (Fig. 12). This resembles the pattern of the epithelial labeling in gingivectomy wounds; ${ }^{17}$ however, the wounds in the gingival crevice are somewhat different from cutaneous or mucosal surface wounds with regards to residual epithelial cells and wound protection so a direct comparison cannot be made relative to the time and pattern of healing of surface and crevicular wounds.

A sharp decline in epithelial labeling was observed in the interval of 24 to 72 hours after scaling and curettage in Zone A with R.A.I. declining from .338 to .028 (Table 1, Fig. 12). The low epithelial labeling in Zone A appeared to be correlated with reestablishment of new epithelial attachment to the tooth surface, and subsequent decrease in irritation and inflammation adjacent to this zone. Normal epithelial attachment was restored in one case as early as five days after the scaling and curettage. This restored epithelial attachment appeared identical to the normal epithelial attachment in newly erupted teeth, described by Engler, Ramfjord and Hiniker, ${ }^{11}$ both with regard to morphology and premitotic labeling of the cells. It seems that the establishment of such an epithelial seal provides a barrier to the spread of bacterial products and irritants into the underlying periodontium. The epithelial cells at the bottom of this seal or attachment apparently do not receive any more stimulus than the epithelial cells in undisturbed Malassez epithelial rests. ${ }^{19}$ Labeling indicating premitotic activity is therefore exceedingly rare in the apical part of the normal epithelial attachment, as it is practically absent in Malassez epithelial rests and in the reduced enamel epithelium prior to eruption. ${ }^{11}$ However, these structures can be stimulated to proliferation as seen associated with inflammation in the immediate vicinity. ${ }^{11,} 19$

It appears from Figure 12 that there is a similarity in the pattern of premitotic activity in the Zones A, B and C following scaling, although both the depressive effect of trauma and the stimulus associated with healing is greater in the crevicular area than at the outer surface of the gingiva.

It is also significant that the labeling of Zone A was considerably less after the new normal epithelial attachment had been established following the scaling than in the control specimens where there still were irritants present in the gingival crevice.

Compared with the post-gingivectomy studies, ${ }^{18,19}$ it apparently took much less time to establish a physiologic gingival crevice following scaling and mild curettage than following a gingivectomy. In no instances folowing gingivectomy was a physiologic gingival crevice established within 21 days after the surgery. But following scaling and curettage a physiologic epithelial attachment appeared as early as five days after the procedure. This fast healing may be due to residual epithelium following the curettage providing multiple "seed areas" for regeneration, and equally important that the tonus of the residual collagenous fibers of the free gingiva restored close adaptation of the gingiva to the tooth surface soon after the injury and thereby enhanced the development of a new epithelial seal. The free gingiva has to regenerate and the collagen to mature before a functional arrangement of the gingival fibers reestablish a physiologic sulcus after gingivectomy. Under the experimental conditions of this study with relatively shallow crevices or pockets not exceeding $4 \mathrm{~mm}$. at the beginning of the treatment, the scaling and light curettage apparently was the preferable treatment 
rather than gingivectomy since the healing was much faster and tissue changes extended to less depth following scaling and curettage than following the gingivectomy.

\section{SUMMARY}

The morphologic patterns and time sequences of healing of the gingival sulcus after scaling and light curettage were studied in three monkeys. The scaling and curettage was performed at various time intervals extending from two hours to thirty-five days before the animals were sacrificed. The three animals all were injected with tritiated thymidine at one hour prior to sacrifice. Radioactive indices were determined by dividing the total number of labeled basal cells by the total number of basal cells for the epithelial attachment, the crevicular epithelium and the gingival surface epithelium.

The epithelial attachment and the epithelial lining of the crevice were not always removed by the scaling and light curettage. The bottom part of the epithelial attachment appeared to be retained more often than the rest of the crevicular lining. The epithelial regeneration reached its peak 1-2 days after the experimental procedure, and continuity of the crevicular epithelium was restored within one and one-half to two days. About 2-3 days after the scaling and curettage, the connective tissue proliferation reached its peak.

A normal epithelial attachment first was observed five days after the experimental procedure. Normal epithelial attachments also appeared in the 2, 3 and 5 week specimens. In the 3 and 5 week specimens, plaque and calculus had started to form on the tooth surface and gingival inflammation was returning in the crevice, although not as yet involving the new epithelial attachment. The R.A.I. of Zone B increased from the 14 day to the 35 day as a result of this new irritation, while the index of Zone A stayed fairly constant, and below the level of the corresponding areas of the nontreated controls.

\section{CONCLUSIONS}

Although the following conclusions apply directly only to rhesus monkeys, there is reason to believe that they in principle apply also to humans:

1. The epithelial regeneration following scaling and gingival curettage occurs mainly from remaining cells of the epithelial attachment and the crevicular lining.

2. The regeneration of epithelium reaches its peak 1-2 days following scaling and curettage.

3. The connective tissue healing is most active 2-3 days following scaling and curettage.

4. A new epithelial attachment may be established as early as 5 days following scaling and curettage.

5. Premitotic labeling is minimal within a normal epithelial attachment, and much less than for any other part of the crevicular and gingival epithelium.

6. Alteration of the normal relationship between the normal epithelial attachment and the tooth, either by instrumentation or by periodontal disease, will be followed by a marked increase in premitotic activity.

7. Normal gingival anatomy and physiology is restored in much shorter time and with considerable less postoperative irritation following scaling and curettage than following gingivectomy, providing that the crevice or pocket is not deeper than 3-4 $\mathrm{mm}$. prior to the treatment.

\section{BIBLIOGRAPHY}

1. Moskow, B. S.: The response of gingival sulcus to instrumentation. A histologic investigation (gingival curettage). J. Periodont., 35:112-125, March-April, 1964.

2. Blass, $J$. L. and Lite, T.: Gingival healing following surgical curettage. A histopathologic study. N. Y. S. Dent. J., 25:127-134, March, 1959.

3. Morris, M. L.: The removal of pocket and attachment epithelium in humans. A histologic study. J. Periodont., $25: 7-11$, Jan., 1954.

4. Moskow, B. S.: The response of gingival sulcus to instrumentation. A histologic investigation in scaling procedure. J. Periodont, 33:282-291, July, 1962. 
5. O'Bannon, J. Y.: Gingival tissue before and after scaling of teeth. $J$. Periodont., 35:69-79, Jan.-Feb., 1965.

6. Ramfjord, S. P. and Kiester, G.: Gingival sulcus and periodontal pockets immediately following scaling of teeth. J. Periodont., 25:167-175, July, 1954.

7. Sato, M.: Histopathological study of the healing process after surgical treatment for alveolar pyorrhea. Tokyo Den. College Bul., 1:71-79, Oct., 1960.

8. Schaffer, E. M., Stende, G. and King, D.: Healing of periodontal pocket tissues following ultrasonic scaling and hand planing. J. Periodont., 35:140-148, Mar.Apr., 1964.

9. Bullough, W. S.: Mitotic activity in the adult female mouse. A study of its relation to the oestrous cycle in normal and abnormal conditions. Philos. Transactions, 231:453-516, May, 1946.

10. Gelfant, S.: Initiation of mitosis in relation to the cell. Div. Cycle Exp. Cell Res., 26:395-403, Mar., 1962.

11. Engler, W. O., Ramfjord, S. P. and Hiniker, J. J.: Development of epithelial attachment and gingival sulcus in rhesus monkey. $J$. Periodont., 36:44-56, Jan.Feb., 1965.

12. Dimassimo, Carmino: Proliferation and migration of cells in the gingival epithelium. Thesis, Univer- sity Rochester, School of Dentistry, $1963,58 \mathrm{pp}$.

13. Messier, G. and LeBlond, C. P.: Cell proliferation and migration as revealed by radioautography after injection of thymidine $\mathrm{H}^{3}$ into male rats and mice. Am. J. Anat, 106:247-285, May, 1960.

14. Lajtha, L. G. and Oliver, R.: The application of autoradiography in the study of nucleic acid metabolism. Lab. Inves., 8:214-224, Jan.-Feb., 1959.

15. Rubini, $J_{\text {., et }}$ a1.: The metabolism and fate of tritiated thymidine in man. J. of Clin. Investig., 39:909. 917, June, 1960

16. Viziam, C. B., Matoltsky, A. G. and Mescon, H.: Enithelization of small wounds. $J$. of Invest. Dermat., 43:499-507, Dec., 1964.

17. Waerhaug, J.: The gingival pocket. Olso. Odontologisk Tidskrif, 1952, 186 pg. (pp. 135-186).

18. Engler, W. O., Ramfjord, S. P. and Hiniker, J. J.: A radioautographic study of healing following simple gingivectomy. I. Epithelization. In press.

19. Ramfjord, S. P., Engler, W. O., and Hiniker, J. J.: A radioautographic study of healing following simple gingivectomy. II. The connective tissue. In press.

20. Bullough, W. S.: The energy relations of mitotic activity. Biol. Revs., 27:133-168, May, 1952.

\section{THE NORTHEASTERN SOCIETY OF PERIODONTISTS}

The Fall Meeting of the Northeastern Society of Periodontists will be held on October 21, 1966 at the Park Sheraton Hotel, New York, N.Y. The speakers who will participate in the program are listed below:

Captain Phillip J. Boyne, DC, USN, Director, Dental Research Department, Naval Medical Research Institute, Bethesda, Maryland

Richard E. Stallard, D.D.S., Ph.D., Chairman, Division of Periodontology, University of Minnesota, School of Dentistry, Minneapolis, Minnesota

D. Walter Cohen, D.D.S., Professor and Chairman, Department of Periodontology, School of Medicine, University of Pennsylvania, Philadelphia, Pennsylvania

Professor Jarmil Kostlan, Director, Institute for Dental Research, Prague, Czechoslovakia

Dr. Max Listgarten, University of Toronto, School of Dentistry, Department of Periodontology, Toronto, Canada

Jerome M. Schweitzer, D.D.S., New York, New York

For further information write Dr. I. W. Scopp, VA Hospital, First Avenue at East 24th Street, New York, New York 10010.

\section{UNIVERSITY OF KENTUCKY}

The University of Kentucky announces the following courses:

November 28, 29, 30, 1966: Periodontics for the General Practitioner, by Drs. Stanley R. Saxe and Harry M. Bohannan and members of the Department of Periodontics. Fee $\$ 100$. Enrollment limited. The primary objective of the course is the presentation of periodontal principles and techniques fundamental to the general practice of dentistry. The program includes a review of the normal periodontium, a detailed discussion of the initiation and progression of periodontal disease and the basic therapeutic procedures applicable by the general practitioner.

March 13 and 14, 1967: Occlusion, Occlusal Disharmonies and the Preservation of the Periodontium, by Dr. Henry Beyron, Stockholm, Sweden. Fee $\$ 60$. Enrollment limited. This course is intended primarily for general practitioners, and will cover how to treat and correct occlusal dysfunction; selective grinding and the use of crowns and bridges in restoring good occlusion, the concept of occlusal relations as part of a harmoniously functioning masticatory system and occlusal dysfunction: its relation to the development and treatment of periodontal disease.

For further information write Dr. Stephen Dachi, Director of Continuing Education, College of Dentistry, University of Kentucky, Lexington, Kentucky. 